\title{
Sentence comprehension and memory for embedded structure
}

\author{
WILLARD LARKIN \\ University of Maryland, College Park, Maryland 20742 \\ and \\ DAVID BURNS \\ The Rockefeller University, New York, New York 10021
}

\begin{abstract}
An experimental simulation technique is introduced to study memory constraints affecting comprehension of embedded sentences. Memory for embedded structure is measured in a pairing task that simulates requirements imposed by sentence comprehension. With increasing memory load (number of embeddings), the rate of performance decline is consistent across all (recall and comprehension) conditions, and reflects a loss of order (as opposed to item) information. Short-term memory overload, rather than linguistic complexity, appears sufficient to account for comprehension difficulty. Performance was imperfect even with singly embedded constructions. Recall showed no abrupt failure with multiple embeddings, indicating that memory constraints are not as discontinuous as English syntax might suggest.
\end{abstract}

Multiply-embedded sentences seem universally troublesome. The difficulty in remembering them was first documented by Miller and Isard (1964), who compared embedded sentences with their right-branching equivalents. There was a sharp decrease in memory performance with more than one embedded clause, precisely the point at which these grammatically possible constructions disappear from ordinary language. Comprehension difficulty follows a similar course, whether it is measured by a paraphrase task (Fodor \& Garrett, 1967), by judgments of grammatical acceptability (Blumenthal, 1966; Marks, 1968), or by tests of reading efficiency (Schlesinger, 1968). Comprehension is not radically enhanced when people are taught the embedded syntax (Stolz, 1967), but performance may improve somewhat if rehearsal time and written aids to memory are provided (Freedle \& Craun, 1970).

These results suggest a crucial role for memory as a limiting factor in comprehension. To clarify this role, it is desirable to assess memory limitations independently of any concomitant linguistic processing which may also impede comprehension. Previous experiments have not been designed to do this. The standard procedures compare performance on embedded sentences with performance on right-branching sentences. For example, in Blaubergs and Braine's (1974) study, subjects were first taught the structure of embedded sentences and then tested for comprehension with an

This research was supported in part by an NIMH predoctoral traineeship and a Maryland Fellowship, awarded to the second author, and by NIMH Grant MH 23878. We thank Carolyn Larkin for help in preparing the stimulus tape and Phyllis Burns for editorial assistance. interrogative probe technique. Performance with embedded sentences was poorer than with right-branching sentences only when there were three or more subjectobject phrases. Although one may account for this result with strong assumptions about short-term memory, embedded sentences differ from other syntactic forms in perceptual and linguistic processing demands as well (Bever, 1970; Holmes, 1973). It is impossible, therefore, to separate the memory constraints in an experiment that simply compares the two syntactic forms.

The following experiment is a study of memory limitations in isolation from other factors that may affect comprehension. Four main conditions are compared: three memory conditions which simulate the task of unraveling embedded syntax but require no comprehension and one baseline condition which measures comprehension difficulty in a customary way. To illustrate how these conditions may be compared, consider the doubly embedded sentence:

\section{(A) The wall that the tapestry that the curator viewed covered crumbled.}

To comprehend $\mathrm{A}$, or any other complex sentence, it is necessary to make the correct links between noun and verb phrases. Noun-verb pairing is not a sufficient condition for comprehension, but without it Sentence A could not be recast as a set of simple propositions:

(B) The curator viewed the tapestry. The tapestry covered the wall. The wall crumbled.

Each sentence in $\mathbf{B}$ joins a noun phrase with a verb 
phrase to express one of the elementary propositions included in $A$. The equivalence of $A$ and $B$ depends uniquely on the noun-verb linkage imposed by the embedded structure. Thus, it is natural to use noun-verb pairing accuracy as a criterion for comprehension in a paraphrase task. Our procedure also uses pairing accuracy as a measure of memory load in a parallel memory task. We view the sequence of nouns and verbs in $A$ as a serial list of items to be held in short-term, or "working," memory. Correct recall (comprehension) of the list (embedded sentence) requires pairing the first with the last item, the second with the next to last item, and so on, as illustrated in Figure 1. A comparison of performance in this memory task with performance in a related comprehension task is our primary interest.

The memory task used three levels of simulation in which properties of embedded sentences were reflected by serial lists. At the first level, sentences were reduced to sequences of nouns and verbs, each sequence having

(a)

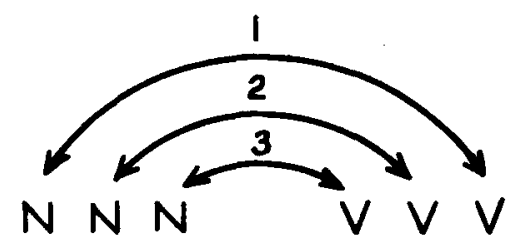

(b)
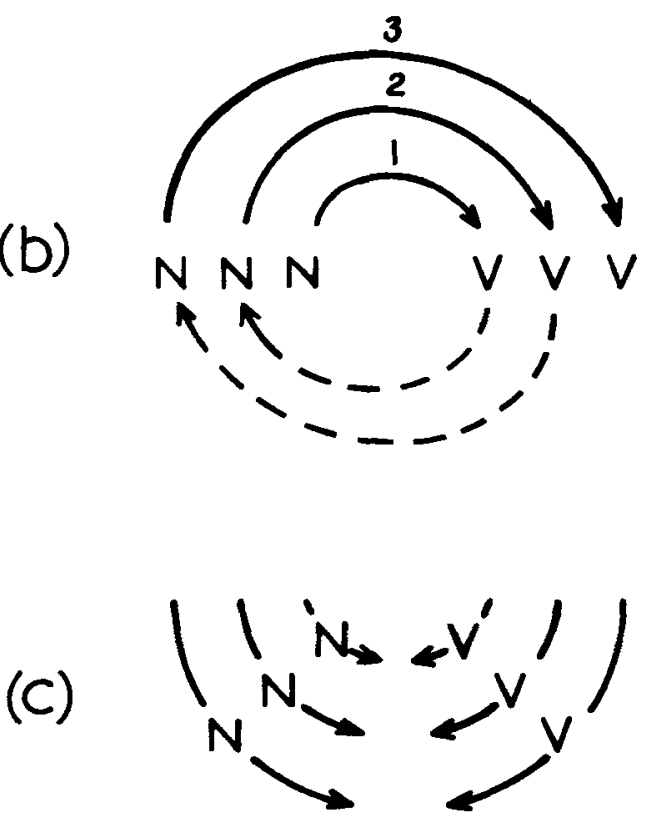

Figure 1. Three schemes for eliminating embedded structure. The letters $\mathbf{N}$ and $\mathrm{V}$ represent nouns and verbs in their respective positions in a sentence with two embeddings. The arrows represent: (a) the "onion skin" strategy, whereby outer pair components are extracted before inner ones; (b) the cyclic approach, which also assigns the correct sequencing of $\mathrm{N}-\mathrm{V} \cdot \mathrm{N}$ triples; (c) the folding scheme, similar to Strategy a, but permits pair extraction without regard to order. the nested structure shown in Figure 1. Second, the nouns were replaced by alphabetic letters and the verbs by digits, thereby breaking the semantic connections between the two halves of each list. Finally digits were used in place of nouns and verbs throughout each list. The subjects' pairing task was unchanged across these three "artificial sentence" conditions.

The instructions for the comprehension task request, but do not require, subjects to complete a linguistic analysis of each sentence as it is presented. As a check on the degree to which these instructions were followed, (1) the degree of semantic constraint on noun-verb relations was varied and (2) a random-words control condition was included, in which the nouns and verbs occurred only in separate lists. Highly constrained semantic relations (as in Sentence A) should facilitate linguistic processing, but should have little effect on short-term memory for noun-verb pairs. If this semantic variable leads to differential performance in the comprehension condition, but not in the noun-verb pairing condition, we will have evidence that the instructions were effective. The random-words condition makes it possible to assess the degree of semantic processing in the noun and verb conditions.

On the basis of memory span data (e.g., Cavanagh, 1972), it may be expected that digit pairing would be superior to letter-digit pairing and that noun-verb pairing would be inferior to both. This hypothesis assumes that the factors governing pairing performance are the same as those governing simple memory span. If, in addition, the pairing task is regarded as a model for sentence comprehension, then memory limitations on noun-verb pairing will define an upper bound for comprehension. Comprehension performance cannot exceed noun-verb pairing unless the basic memory limitations are compensated by perceptual or linguistic factors that can operate at the single-sentence level.

The relation between pairing performance and comprehension becomes more complex under hypotheses that attribute difficulty with multiply embedded structure to specific deficiencies in linguistic analysis, rather than to memory (e.g., Bever, 1970; Holmes, 1973). As the number of embeddings increases, linguistic burdens accumulate faster than the additional demand on memory. These burdens vanish for singly embedded syntax. As a result, comprehension and pairing performance may not be parallel, or one may not strictly dominate the other.

Previous studies have shown a substantial decrease in the comprehensibility of embedded sentences as their length (number of embeddings) increases. If memory alone is responsible, as Bar-Hillel (1964), Chomsky (1953), Deese (1970), and others have proposed, then a similar performance pattern should be observed in the pairing tasks: Our sharply limited linguistic capacity could then be understood as a symptom of a more general constraint. 


\section{METHOD}

\section{Subjects}

Seventy-nine University of Maryland students participated in individual $1-\mathrm{h}$ sessions. In Conditions 1 through 4 , there were $15,16,17$, and 17 subjects, respectively. The remaining 16 served in a random-words control condition related to Condition 3.

\section{Procedure}

Subjects read the instructions, then listened to them repeated over headphones while seated in a soundproof enclosure. Instructions and stimuli were presented monaurally to the right (left) ear for right- (left-) handed subjects.

In the pairing tasks, the stimuli were digits (Condition 1), letters followed by digits (Condition 2), or nouns followed by verbs (Condition 3). An expert speaker recorded these materials on tape in a monotone voice, at a rate of about $1 \mathrm{item} / \mathrm{sec}$. On each trial, 4, 6, 8, or 10 items were presented. Two to $16 \mathrm{sec}$ were allotted for responses between trials.

A $1-\mathrm{kHz} 250-\mathrm{msec}$ tone was interposed to mark the midpoint of each stimulus list. This marker served to equalize uncertainty about list length across conditions. It was the only cue to list length in Condition 1 , but it was a redundant cue in Conditions 2 and 3 , as these lists had midpoint transitions from letters to digits or from nouns to verbs.

The digits for Condition 1 were selected randomly without replacement from the set 0 to 9 . For Condition 2, the set was 1 to 9 . Letters for Condition 2 were sampled randomly without replacement from the set $F, G, H, J, L, N, T, W, Y$ (chosen to minimize acoustic confusions). Obvious patterns and acronyms were eliminated. The letter set was printed on a display card in the subject's booth.

Nouns and verbs for Condition 3 were extracted in sequence from the embedded sentences of Condition 4, except that terminal object nouns were omitted when necessary. The same nouns and verbs were used in the random-words control condition. Homogeneous noun lists and verb lists were constructed for the control condition by sampling from fixed-length stimulus sequences in Condition 3 , and randomly assigning the words to noun lists or to verb lists of the same length.

Embedded sentences for Condition 4 were recorded at a normal conversational rate, in a highly expressive voice. No midpoint marker tone was used. Each sentence was constructed by prefacing a noun-verb list with "The," and inserting "that the" between adjacent nouns. Noun-verb pacing and trial timing closely matched the other conditions.

\section{Instructions}

Subjects in the pairing tasks (Conditions 1, 2, 3 and control) were instructed to recall the items in ordered pairs; first item paired with last, second item paired with next to last, and so on. The pairs could be recited in any sequence, but the ordering of items within each pair had to match the temporal ordering of items in the stimulus list. A "folding diagram" similar to Figure 1c was used in the printed instructions, along with suggestions for systematic approaches to the task.

In Condition 4, subjects were asked to comprehend, and then to express, each embedded sentence as a set of simple sentences having the same meaning as the original. Subjects could abbreviate the simple sentences by omitting connectives and object nouns. With these omissions, the response requirements of the comprehension task matched those of the pairing tasks. The desired simple sentences were illustrated for four embedded sentence examples, one at each length (just as Sentence A was illustrated earlier in this paper). Freedle \& Craun's (1970) nested propositions were used as examples.

\section{Sentences}

Embedded sentences for Condition 4 had noun-verb relations which were either semantically neutral or partially constrained.
The neutral sentences were of two types, denoted ND (ending in a direct object) or NR (ending in a reflexive verb). The sentences were constructed with nouns and verbs that were compatible in all possible combinations of subject, action, and object. In neutral sentences (for example, "The testimony that the sermon quoted distorted the lesson"), nouns and verbs could be permuted without risk of semantic anomaly or loss of grammaticality. (One permuted version of the example would be, "The sermon that the testimony distorted quoted the lesson.") In Type NR sentences, semantic neutrality required special dualfunction verbs which could serve as the nucleus of a subjectaction-object triple (e.g., "defenders withdrew army") or as the terminal member of a subject-action pair ("defenders withdrew" or "army withdrew"). Verbs with flexibility in these transitive and reflexive roles do not seem plentiful in English. We know of no compilation of them other than the list given in the Appendix.

The ND and NR sentences were used to insure comparability between the abstract (digit or letter-digit) pairing tasks and the word-pairing or comprehension tasks. If certain verbs could not take dual syntactic roles, or if some noun-verb combinations violated conventional usage, the sentences were designated Type C (semantically constrained). Type $C$ sentences varied in the degree to which semantic relations provided a basis for nounverb pairing or for comprehension. Sentence A, considered earlier, is an example of Type $C$ with fairly strong constraints. The three types of sentences, ND, NR, and C, were presented equally often in a random sequence.

\section{RESULTS}

The two dependent measures of interest were (a) the numbers of pairs recalled which were correctly joined and (b) a measure of pairing efficiency, defined as twice the number of pairs correctly joined divided by the number of items recalled. The efficiency measure represents an attempt to control for the different number of items recalled across list length. If no pairing errors are made, the efficiency score will be $1(100 \%$ efficiency), regardless of the number of items recalled. If additional unpaired items are recalled, or if some items are incorrectly paired, the ratio of pairs to items drops below $2: 1$, and the efficiency score drops accordingly. Measures $a$ and $b$ gave very similar results.

Table 1

Pairing Performance*

\begin{tabular}{llllll}
\hline \multirow{2}{*}{ Condition } & & \multicolumn{4}{c}{$\begin{array}{c}\text { Length of Stimulus } \\
\text { (Number of Items) }\end{array}$} \\
\cline { 3 - 6 } 1 Digits & & 4 & 6 & 8 & 10 \\
\hline \multirow{3}{*}{ 2 Letters and Digits } & Pairs & 1.89 & 2.26 & 1.82 & 1.48 \\
& Efficiency & $(.95)$ & $(.84)$ & $(.72)$ & $(.57)$ \\
3 Nouns and Verbs & Pairs & 1.80 & 2.07 & 1.68 & 1.46 \\
& Efficiency & $(.91)$ & $(.78)$ & $(.61)$ & $(.51)$ \\
4 4 Sentences & Pairs & 1.75 & 1.47 & 1.13 & 1.05 \\
& Efficiency & $(.89)$ & $(.62)$ & $(.51)$ & $(.47)$ \\
& Pairs & 1.69 & 1.25 & 1.09 & 1.04 \\
& Efficiency & $(.87)$ & $(.55)$ & $(.52)$ & $(.51)$ \\
\hline
\end{tabular}

* Upper entries are the mean number of pairs correctly reported at each presentation length: The variance of this measure among subjects is less than .25 for each entry. Lower entries are efficiency scores: $2 \times$ (Number of Correct Pairs)/(Number of Correct Items). 


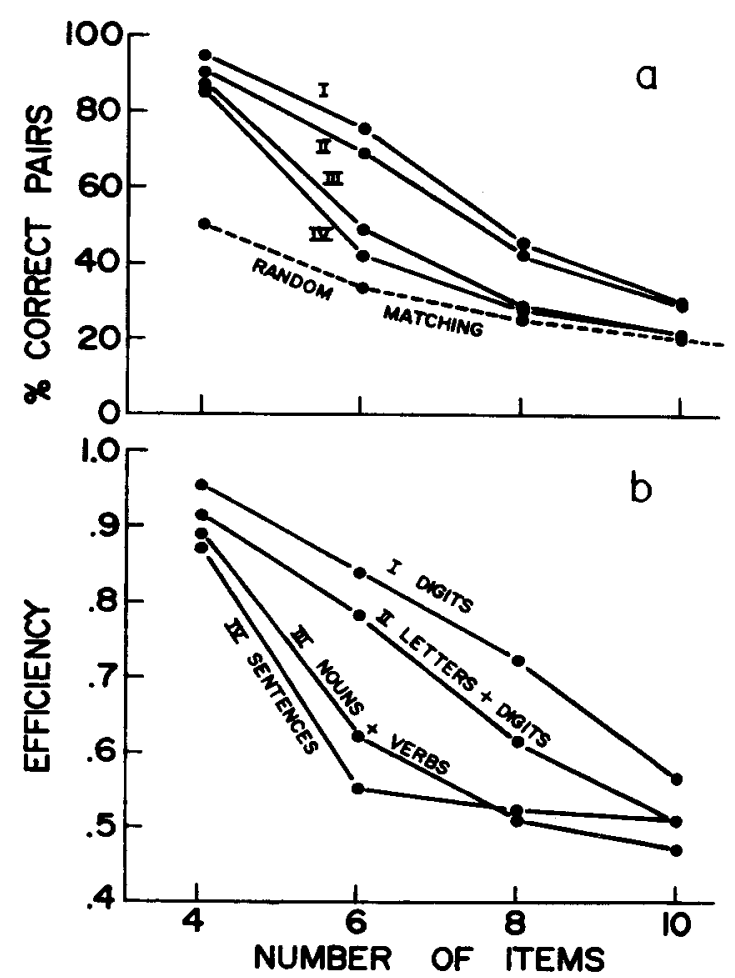

Figure 2. Pairing performance in each condition as a function of list (or sentence) length.

Consequently, while both are summarized in Table 1 and plotted in Figure 2, we report statistics only for the number of pairs correctly joined.

The initial 12 trials in each session were discarded, and the remaining 60 trials showed no discemible effects of practice. Averaged over conditions, the number of correct pairings decreased significantly $[\mathrm{F}(3,144)=35.7$, $\mathrm{MSe}=.108, \mathrm{p}<.001]^{1}$, from a maximum near two for the four-item lists to nearly chance performance for longer lists. The "chance performance level," indicated by the dashed line in Figure 2a, represents expected performance assuming that items are matched at random. Because the expected number of correct matches based on random pairing behavior is independent of the number of items to be matched (Mosteller \& Bush, 1954), this baseline does not depend on intertrial or intersubject differences in the short-term retention of stimulus materials.

The overall differences among conditions were significant $[\mathrm{F}(3,48)=8.58, \mathrm{MSe}=.468, \mathrm{p}<.001]$, and there was no significant interaction of Condition by List Length. Although the conditions were ordered consistently across list lengths, Conditions 1 and 2 were not significantly different, nor were Conditions 3 and 4 . Performance in the former two (abstract) conditions was superior to that in the latter two (linguistic) conditions $[\mathrm{F}(1,60)=41, \mathrm{MSe}=.454, \mathrm{p}<.001]$.

To determine the effect of semantic constraint, a separate analysis of variance was performed for the data from Conditions 3 and 4 , with both list length and semantic constraint (sentence type) included as independent variables. There were significant overall differences among list lengths $[F(3,96)=52.3, \quad M S e=.020$, $\mathrm{p}<.001]$ and among levels of semantic constraint $[\mathrm{F}(2,64)=3.9, \mathrm{MSe}=.124, \mathrm{p}<.05]$. The interaction of List Length by Semantic Constraint was significant $[\mathrm{F}(6,192)=5.2, \mathrm{MSe}=.107, \mathrm{p}<.01]$. The interaction of Conditions by List Length was not significant, and the differences between Conditions 3 and 4 were insignificant at every list length when multiple $t$ tests were applied.

A post hoc analysis of the 24 means involved in the third-order interaction of Condition ( 3 and 4 ) by Length by Semantic Constraint showed that semantic variation had a significant effect only for Sentence Length 6 (three pairs) in Condition 4. In particular, the unconstrained Type ND and NR sentences were processed less accurately than Type $\mathrm{C}$ sentences (difference in means $=.576$, Tukey HSD $=1.65$, $\mathrm{p}<.05$ ). Differences for the other sentence lengths were in the same direction, but did not reach statistical significance. Semantic constraint had no effect in Condition 3, and Type $\mathrm{C}$ sentences did not differ from the corresponding noun-verb pairs for any list length.

The random-words control condition confirmed the absence of a general semantic facilitation in Condition 3 . Although noun pairs were recalled with slightly greater accuracy than verb pairs, the combined results for random-word lists closely overlapped the Condition 3 data. A detailed presentation of these control data has been omitted.

The response protocols gave clear evidence of memory limitations. Subjects often attempted first to pair the initial and terminal items (i.e., the exterior pair) or the two adjacent items in the center of a list. Other items were recalled later in the response period, and were paired with greater hesitation. The outermost pairs of the "folded" pair sequence (Figure 1c) were recalled with greater frequency and accuracy than the interior pairs. Thus, the serial position curves were U-shaped over the pair positions and $\mathrm{W}$-shaped over the item positions.

\section{DISCUSSION}

Performance in the four main conditions is ordered as one would expect on the basis of memory span results for similar stimuli (Cavanagh, 1972). The constant rate of performance decline (no Condition by Length interaction) indicates the primary limitation of immediate memory on pairing performance. The claim of these stimulus materials upon memory capacity was not offset by the categorical distinctions between first-half and second-half items (which would favor Condition 2 over Condition 1), nor by the semantic relations in the nounverb pairs (which would favor Condition 3 over Condition 2). This strong dependence on stimulus materials shows that pairing performance is a rather 
direct measure of memory load: The mental operations needed to unravel embedded structure tax the same limited capacity as do the items in a simple memory task.

The digit and letter-digit pairing data allow two conclusions. First, the rapid decline in pairing accuracy with increasing length shows that difficulty with embedded structure is not confined to a linguistic context. Although pairing performance is superior in these abstract conditions, the rate of decline is comparable across all four conditions. Short-term "working" memory was implicated in this performance decline by our subjects' voiced impressions that stimulus items seemed to be "erased" before the embedded structure could be fully processed.

Second, the smooth decline in pairing efficiency for abstract material (Figure 2) suggests that memory processes do not encounter any special discontinuities as the depth of embedding increases. Instead, the number of correct pairs actually increases slightly, reaching a maximum for the six-item sequences (Table 1). If multiple embeddings were completely incompatible with short-term memory abilities, we should have seen a sharp break, or discontinuity, rather than a steady decline in pairing efficiency. As we have noted, the memory load is severe, but the degree of difficulty makes no sudden acceleration in the critical region between single and double embeddings.

A smooth decline in pairing performance is less obvious for Conditions 3 and 4 . Nevertheless, the absence of a Condition by Length interaction (for either the pairing or the efficiency measure) suggests that the rate of decline may be nearly the same for words and sentences as it is for the abstract materials. The hypothesis that multiple embeddings pose greater obstacles for sentence comprehension than for word pairing is refuted by the lack of differences between Conditions 3 and 4 . This conclusion is strengthened by the extreme steps (i.e., multiple $t$ tests) taken to reveal such differences.

The correspondence between Conditions 3 and 4 lends credence to the noun-verb pairing scheme as a rudimentary model for sentence comprehension. This interpretation is limited, of course, by the possibility that subjects used other than normal linguistic strategies in Condition 4. Our attempt to induce a normal comprehension strategy depended heavily on the subjects' commitment to instructions. This commitment could not be measured directly, but it was enough to produce a detectable difference between Conditions 3 and 4 in the extent to which subjects used semantic information. The superior performance with semantically constrained sentences is evidence that some normal comprehension skills were engaged in Condition 4, notwithstanding the artificial laboratory context. In contrast, Condition 3 performance showed no sensitivity to semantic constraints. The absence of semantic facilitation in Condition 3 is also underscored by the random- words data, which were indistinguishable from the Condition 3 results.

The effect of semantic constraint, nevertheless, was marginal. Because our primary concern was to equate the various pairing tasks, and to make them comparable with the sentence task, the amount of constraint was kept relatively low. All of the sentences were constructed so that permutations of noun-verb relationships would have little effect on interpretability. Semantically constrained sentences had fewer interchangeable components than did neutral sentences, but semantic relations alone were never sufficient to decode the embedded syntax. The restricted range of semantic constraint probably accounts for the small effects observed.

The ability to recall multiply embedded lists or sentences was severely taxed in all of the experimental conditions. It seems clear that the mental reordering operation, rather than the length of the stimulus sequences, was the main burden on subjects' processing capacity. The results for singly embedded lists and sentences confirm this interpretation. For these (short) stimulus presentations, the immediate memory span exceeds the stimulus length, but the pairing task remains quite difficult. Individual items in short lists were almost always recalled, but response errors were introduced by interchanging the item relationships. In other studies of short-term memory, Estes (1972) has observed that positional information may not be as well encoded as item information. Our data reinforce this observation. We find it striking that nearly $15 \%$ of the singly embedded sentences were incorrectly interpreted. If, in normal English discourse, people deal successfully with singly embedded constructions, they must rely upon more than the memory-pairing operation isolated in these experiments. The contextual cues, social expectations, and rhythmic stress patterns usually regarded as mere facilitating factors may be indispensible in normal discourse.

Our technique of experimental simulation relies on a simple, but not wholly insufficient, model for processing syntax in memory. The pairing model leads to the expectation that memory demands are accessible in relative isolation from linguistic capacities. A similar technique may be useful in other areas. For example, consider Kintsch's (1974) idea that comprehension depends upon constructing a "text base," that is, a structured list of propositions and their interrelations. For Kintsch, comprehension entails a "natural" retrieval scheme for accessing individual propositions, whereas memory for unrelated words requires construction of an "unnatural" scheme for relating the list items. A text base should be more easily established in the "natural" case. Then, if both items and scoring procedure are matched, memory for text should be more efficient than memory for unrelated items. This advantage did not appear in the present data, probably because individual sentences were studied in isolation from meaningful context. Our data 
are consistent with the notion that a more elaborate semantic context may be necessary for good comprehension, and Kintsch (1974) considers deep semantic processing essential to his model. The memory simulation technique, generalized to the problem of recall of text, could provide important information about memory storage and retrieval in Kintsch's theory. The technique worked well enough to suggest its use in the study of other complex cognitive operations (e.g., mental arithmetic, concept identification, or logical reasoning) in which short-term memory also plays a dominant role.

\section{APPENDIX}

Conditions 3 and 4 were based in part upon semantically neutral sentences ending in dual-function verbs. These sentences (Type NR) are given below in the format for Condition 3. Full sentences were formed by prefacing the first noun with "The" and inserting "that the" between adjacent nouns. Materials not used for data analysis are omitted.

1. reaction bacteria weakened survived

2. army defenders withdrew stopped

3. meeting union organized disbanded

4. clown seal begged applauded

5. boy farmer answered refused

6. clerk customer boy left answered tripped

7. shaft cable machine broke held pulled

8. watchman operator police awakened telephoned answered

9. nurse doctor parents watched worried stopped

10. device clock switch locked stopped broke

11. witness girl clerk victim stopped fought remembered left

12. hunter spy prisoner guard hid left watched passed

13. truck convoy traffic soldiers detoured trailed passed stopped

14. audience doorman escort usher approached hurried followed left

15. team coach gymnast crowd applauded worried stopped failed

16. athlete coach owner cashier man struck assisted approached searched attacked

17. trainer jockey agent owner promoter joined succeeded bet worried talked

18. policeman detective thief deputy informant obeyed assisted paid searched worried

19. boatman messenger captive guard officer tripped hid revived succeeded drowned

20. operator doorman maid actor critic remembered married puzzled assisted departed

\section{REFERENCES}

Bar-Hillel, Y. Language and information: Selected essays on their theory and application. Reading, Mass: AddisonWesley, 1964.
Bever, T. G. The influence of speech performance on linguistic structures. In G. B. Fiores D'Arcais \& W. J. M. Levelt (Eds.), Advances in psycholinguistics. Amsterdam: North-Holland, 1970.

Blaubergs, M. S., \& Braine, M. D. S. Short-term memory limitations on decoding self-embedded sentences. Journal of Experimental Psychology, 1974, 102, 745-748.

Blumenthal, A. L. Observations with self-embedded sentences. Psychonomic Science, 1966, 6, 453-454.

Cavanagh, J. P. Relation between the immediate memory span and the memory search rate. Psychological Review, 1972, 79, 525-530.

Сномsку, $\mathbf{N}$. On certain formal properties of grammars. Information and Control, 1953, 2, 133-167.

DeEse. J. Psycholinguistics. Boston: Allyn \& Bacon, 1970.

Estes, W. K. An associative basis for coding and organization in memory. In A. W. Melton \& E. Martin (Eds.). Coding processes in human memory. Washington, D.C: V. H. Winston, 1972.

Fodor, J. A., \& GARRETT, M. Some syntactic determinants of sentential complexity. Perception \& Psychophysics. 1967. 2. $289-296$.

Freedle, R., \& Craun, M. Observations with self-embedded sentences using written aids. Perception \& Psychophysics, 1970, 7, 247-249.

Holmes. V. M. Order of main and subordinate clauses in sentence perception. Journal of Verbal Learning and Verbal Behavior, 1973, 12, 285-293.

KINTsCH, W. The representation of meaning in memory. New York: John Wiley, 1974.

MarKs, L. E. Scaling of grammaticalness of self-embedded English sentences. Joumal of Verbal Learning and Verbal Behavior, 1968, 7, 965-967.

Miller, G. A., \& IsARD, S. Free recall of self-embedded English sentences. Information and Control, 1964. 7. 292-303.

Mosteller, F., \& Bush, R. R. Selected quantitative techniques. In G. Lindzey (Ed.), Handbook of social psychology (Vol. 1). Reading. Mass: Addison-Wesley, 1954.

SCHLEsinger, I. M. Sentence structure and the reading process. The Hague: Mouton, 1968.

STolz, W. S. A study of the ability to decode grammatically novel sentences. Journal of Verbal Learning and Verbal Behavior, 1967. 6, 867-873.

\section{NOTE}

1. The mean squared error (MSe) given for each $F$ statistic estimates the average error variance per subject trial. A few subjects were randomly eliminated from some analyses to satisfy sample size requirements of the computer program.

(Received for publication March 19. 1976; revision received June 9,1976 .) 